\section{Aglomerados ativos de COVID-19 em Santa Catarina, Brasil, e tendência de mobilidade dos locais de trabalho}

\author{
Active COVID-19 clusters in Santa Catarina, Brazil, \\ and workplace mobility trends
}

\author{
Aglomerados activos de COVID-19 en Santa \\ Catarina, Brasil, y tendencia de movilidad \\ en lugares de trabajo
}

\author{
Ivan Merêncio 1,2 \\ Gecielli Martins Monteiro ${ }^{3}$ \\ Carlos Antônio Oliveira Vieira ${ }^{1}$
}

doi: 10.1590/0102-311X00301620

\section{Resumo}

A aplicação da análise espacial destinada ao estudo de dados epidemiológicos humanos se tornou notória nas últimas duas décadas. Nesse sentido, este artigo aborda a estatística scan para a detecção de clusters espaço-temporais de casos da COVID-19 em Santa Catarina, Brasil. O objetivo é aplicar a estatística scan para a identificação de agrupamentos ativos, determinando sua localização, dimensão e ordem (prioridade). A organização da base descritiva abrangeu os casos de COVID-19 entre 1o de março e 31 de agosto de 2020, disponíveis no Portal de Dados Abertos do Estado de Santa Catarina. A base vetorial dos limites municipais e mesorregiões catarinenses, e as populações estimadas para 2020 foram obtidas no site do Instituto Brasileiro de Geografia e Estatística (IBGE). A covariável tendência de mobilidade dos locais de trabalho foi obtida no documento COVID-19: Relatório de Mobilidade da Comunidade do Google. Para a execução da estatística, considerou-se o modelo discreto de Poisson, apoiado na abordagem prospectiva. No resultado do trabalho, evidenciou-se a capacidade do procedimento para delimitação dos clusters, o qual identificou 17 clusters ativos com a variável resposta e 18 ativos após a inclusão da covariável, distribuídos em todo estado, predominantes no litoral e no Oeste Catarinense. O cluster primário localizou-se no Sul Catarinense. A covariável tendência de mobilidade dos locais de trabalho influenciou moderadamente em 38,89\% dos aglomerados. O método foi eficiente para a compreensão da distribuição espacial da epidemia. Isso caracteriza a estatística scan como uma ferramenta de apoio a execução de ações a serem tomadas por gestores, priorizando áreas mais afetadas pela doença.

\author{
Correspondência \\ I. Merêncio \\ Rua Vinte e Três 420, Meleiro, Santa Catarina 88920-000, \\ Brasil. \\ ivan.merencio@posgrad.ufsc.br \\ 1 Universidade Federal de Santa Catarina, Florianópolis, Brasil. \\ 2 Universidade do Extremo Sul Catarinense, Criciúma, Brasil. \\ 3 Universidade Nove de Julho, Guarulhos, Brasil.
}




\section{Introdução}

O primeiro surto da doença COVID-19, causada pelo novo coronavírus da síndrome respiratória aguda grave 2 (SARS-CoV-2), ocorreu em dezembro de 2019 na cidade de Wuhan, na China 1,2. Em pouco tempo, o vírus se disseminou no país, apresentando uma dispersão quase exponencial 3. Em 11 de março de 2020, a Organização Mundial da Saúde (OMS) definiu a COVID-19 como uma pandemia, devido à ocorrência de casos confirmados da doença em todos os continentes do planeta 4.

Geralmente, a transmissão ocorre pelo contato direto ou indireto com as secreções (gotículas respiratórias) de pessoas infectadas, que são expelidas durante a tosse, espirro ou fala 5.

Diante dos estágios iniciais da campanha de vacinação em massa da população brasileira, evitar a exposição ao vírus ainda é a forma mais eficiente de prevenção ${ }^{3}$. Distanciamento social, isolamento dos casos confirmados, acompanhamento dos doentes e medidas de higiene são as principais ações adotadas para evitar a disseminação do vírus 6 .

Até o dia 29 de agosto de 2020, o Brasil contabilizava 3.846 .153 casos e 120.462 óbitos confirmados de COVID-19, com taxa de letalidade de 3,13\%. A Região Sul do país computava o total de 399.787 infectados e 8.837 mortes, com taxa de letalidade de 2,21\% 7 .

A empresa Google disponibilizou na Internet o documento denominado COVID-19: Relatórios de Mobilidade da Comunidade (https://support.google.com/covid19-mobility/answer/9824897?hl= pt-BR\&ref_topic=9822927, acessado em 22/Ago/2020), visando auxiliar os órgãos competentes nas estratégias de enfrentamento à COVID-19. Esses relatórios fornecem registros anonimizados das tendências de movimentação por localização geográfica ao longo do tempo, em diversas categorias de locais, como lazer, comércios, locais de trabalho, residências, entre outros. Os registros não apontam números absolutos, mas, sim, taxas relativas a um valor de referência, que é a mediana do período de cinco semanas de janeiro 2020. Para a utilização desses índices, é necessário realizar cinco etapas de verificação que têm por objetivo garantir a calibração dos dados da região analisada.

Em panoramas de surtos e epidemias, a análise espacial é uma ferramenta que possibilita a identificação de áreas de maior risco ${ }^{8}$. A estatística scan (ou de varredura), originalmente apresentada por Kulldorff et al. 9 , em 2005, destina-se à avaliação estatística do objeto de estudo no espaço geográfico, com o intuito de detectar e hierarquizar clusters de regiões que demandam maior atenção.

A estatística scan busca detectar uma região composta por clusters, comparando o número de casos observados em relação aos esperados. Esses aglomerados são constituídos por meio da modificação do raio, que varia de zero até o valor máximo de $50 \%$ da população no interior do cluster ${ }^{10}$. Determina-se a razão entre a verossimilhança com a hipótese alternativa de que o risco de acontecimentos do evento é maior dentro do que fora do cilindro. A hipótese nula é de que a divergência entre as duas verossimilhanças ocorra ao acaso 11 .

Existem duas abordagens temporais que podem ser empregadas na estatística scan: a retrospectiva e a prospectiva. A análise retrospectiva tem o intuito de detectar tanto conglomerados que deixaram de existir antes do término do estudo, denominados clusters históricos, quanto de conglomerados em atividade até o fim da pesquisa, chamados de clusters ativos. Por sua vez, a análise prospectiva preocupa-se apenas com a identificação de agrupamentos ativos.

O método pode ser utilizado em diferentes áreas, como na astronomia, geografia, zoologia e em estudos epidemiológicos para a busca de aglomerados de doenças. Como exemplo, pode-se citar o monitoramento de agrupamentos ativos da COVID-19 no território brasileiro, evidenciando as localidades que apresentaram excesso de casos e óbitos para a doença no período de 25 de fevereiro a 1o de maio de 2020, ou seja, estágios iniciais da pandemia no país 12.

Assim, considerando o presente momento da COVID-19 em Santa Catarina, Brasil, este artigo tem como objetivo aplicar a estatística scan para detectar agrupamentos ativos da doença no estado ao nível de 5\% de significância, classificando-os conforme a prioridade de intervenção. 


\section{Método}

\section{Caracterização da área de estudo}

Trata-se de estudo ecológico, cuja área de análise foi o Estado de Santa Catarina, que está situado na Região Sul do Brasil e faz limite com os estados do Rio Grande do Sul e do Paraná, como também com o Oceano Atlântico e a Argentina. Tem 295 municípios, com população estimada de 7.252.502 habitantes, e ocupa área de $95.730,684 \mathrm{~km}^{2}$. A Rodovia BR-101 é o principal eixo de ligação com as demais regiões da federação (Instituto Brasileiro de Geografia e Estatística. Cidades@. https://www. ibge.gov.br/cidades-e-estados/sc, acessado em 22/Ago/2020).

As unidades territoriais de estudos foram os municípios. A apresentação dos resultados obedeceu à classificação das mesorregiões do estado: Grande Florianópolis, Norte Catarinense, Oeste Catarinense, Serrana, Sul Catarinense e Vale do Itajaí.

\section{Materiais}

Para a análise espaço-temporal da COVID-19 em Santa Catarina, utilizou-se pesquisa bibliográfica e documental. Os registros das ocorrências confirmadas sobre o coronavírus do período de 1o de março de 2020 a 31 de agosto de 2020 foram extraídos do boletim epidemiológico estadual disponível no Portal de Dados Abertos do Estado de Santa Catarina (Dados anonimizados: casos confirmados COVID-19. http://www.dados.sc.gov.br/dataset/covid-19-dados-anonimizados-de-casos-confir mados/resource/76d6dfe8-7fe9-45c1-95f4-cab971803d49, acessado em 04/Set/2020). O arquivo de acesso público, com extensão CSV, estava organizado por municípios e continha as informações relacionadas aos infectados pela doença.

Essa série de dados englobou os casos confirmados de COVID-19 que apresentaram síndrome gripal, síndrome respiratória aguda grave (SRAG) ou assintomáticos, cujos casos foram definidos por critério clínico-epidemiológico, laboratorial ou não informado. O diagnóstico foi realizado pelos métodos RT-PCR, por testes imunológicos rápidos, ambos ou não informado.

A tendência de mobilidade dos locais de trabalho das principais cidades foi obtida no relatório de mobilidade do Google (COVID-19: relatórios de mobilidade da comunidade. https://www. google.com.br/covid19/mobility/, acessado em 22/Ago/2020), disponível pela empresa na Internet no formato .csv.

A base vetorial contendo os limites municipais e das mesorregiões catarinenses do ano de 2010 foi disponibilizada pelo Instituto Brasileiro de Geografia e Estatística (IBGE) em seu portal (Malha municipal. Downloads: municipio_2019. https://www.ibge.gov.br/geociencias/organizacao-doterritorio/15774-malhas.html?=\&t=downloads, acessado em 22/Ago/2020), em formato Shapefile, datum SIRGAS 2000 e em coordenadas geodésicas. O número estimado de habitantes dos munícipios para o ano de 2020 também foi originado pelo IBGE (Estimativas de população 2020. https://www. ibge.gov.br/estatisticas/sociais/populacao/9103-estimativas-de-populacao.html? =\&t=downloads, acessado em 22/Ago/2020).

\section{Estatística scan: modelo discreto de Poisson}

Essa etapa iniciou com a sistematização dos dados descritivos no programa LibreOffice Calc (https:// pt-br.libreoffice.org). Os registros essenciais do boletim epidemiológico para o trabalho foram: identificador do caso (gerado pelos autores), data provável de início dos sintomas e coordenadas geodésicas do centroide da sede municipal de residência. Na sequência, excluíram-se 5.831 infectados oriundos de outros estados ou países, pois não era possível localizá-los na base cartográfica devido à ausência de informações.

Diversos estudos evidenciaram a redução dos impactos da pandemia de COVID-19 com as medidas de distanciamento social 6,13. Desse modo, optou-se pela inclusão de uma covariável que atendesse essa característica. Para o Estado de Santa Catarina, o conjunto de dados que estava mais completo era a tendência de mobilidade dos locais de trabalho, ou seja, a taxa relativa de trabalho presencial; portanto esta foi definida como a covariável de estudo. As taxas das principais cidades de cada mesor- 
região foram adotadas como referência para a sua localidade. Os munícipios de Alfredo Wagner, Ituporanga e Joaçaba apresentaram ausência de registros em algumas datas, que foram completados com o índice médio de Santa Catarina.

Para a finalização da base descritiva, foi feita a união entre os casos, população municipal e covariável. Esse arquivo foi salvo no formato DBF.

Para verificar a existência de conglomerados, utilizou-se o software SaTScan 9.6 (http: \\www. satscan.org), apoiado no modelo discreto de Poisson 14, pois o boletim era composto por uma contagem de casos da COVID-19, e a população exposta ao risco varia conforme o munícipio, ou seja, o número esperado de infectados é proporcional ao tamanho de sua população.

Nesse sentido, a variável resposta foi o número de casos confirmados de COVID-19 em Santa Catarina, que são dados pontuais com centroides localizados nas sedes dos municípios catarinenses no intervalo de 1o de março de 2020 a 31 de agosto de 2020 .

Há possibilidade de inclusão de covariáveis para a explicação de um conglomerado ou parte dele, que se confirma quando este desaparece ou diminui de tamanho durante as análises. Porém pode suceder, com a inclusão da covariável, o surgimento de um aglomerado significativo que não havia aparecido antes no modelo ajustado. Isso ocorre quando a distribuição espaço-temporal da covariável camufla o conglomerado 15 .

O SaTScan 9.6 exige que as covariáveis sejam representadas por números inteiros no formato qualitativo ordinal. Assim, foi necessário categorizar a tendência de mobilidade dos locais de trabalho. Para tanto organizou-se a tendência de mobilidade em seis classes, sendo 1 a que corresponde à maior taxa de trabalho presencial e 6 à menor: 1 (> 13\%); 2 (12\% a -6\%); 3 (-7\% a $-25 \%) ; 4(-26 \%$ a $-44 \%)$; $5(-45 \%$ a $-63 \%)$ e $6(<-64 \%)$.

A estatística scan atua com a varredura de diversos raios de busca, por isso foi necessário definir esse limite. Assim, estipulou-se como parâmetro máximo de detecção espacial o valor de 50km, com o objetivo de evitar que um único cluster englobasse várias mesorregiões.

Determinou-se como limite temporal máximo a porcentagem de $50 \%$ do total de infectados no interior do cluster, com o intuito de identificar a data de início dos conglomerados.

A busca de conglomerados ativos foi baseada na inicialmente na análise prospectiva sem covariável e, posteriormente, com o uso da covariável. Os agrupamentos ativos são aqueles que existiram até fim do estudo. Além disso, definiu-se o modo de busca sem a sobreposição de aglomerados.

Os limites territoriais do Estado de Santa Catarina foram limitantes para a identificação de aglomerados nas regiões fronteiriças, pois a análise foi restrita aos dados dos residentes no estado.

Para cada suposto aglomerado, foi calculado o valor da razão de verossimilhança generalizada de Poisson (RVG), e aquele que apresentasse o maior valor era o mais provável, denominado de primário. Para o teste da significância desse conglomerado primário, considerou-se $5 \%$ de probabilidade com as seguintes hipóteses: (i) H0: não existe agrupamento espaço-temporal de COVID-19 em Santa Catarina; (ii) H1: existe agrupamento espaço-temporal de COVID-19 em Santa Catarina.

O procedimento de Monte Carlo foi empregado para testar essas hipóteses com relação ao conglomerado primário. E, após as 999 permutações, se a ordem R estivesse acima de 950, contatava-se que o agrupamento das ocorrências naquele conglomerado não aconteceu devido ao acaso. Os clusters com valores de RVG menores, ditos secundários, tiveram avaliação da significância de modo similar ao realizado no primário.

Por fim, utilizou-se o software QGIS 3.10 .8 (https://qgis.org/en/site/) para espacialização dos resultados obtidos no SaTScan 9.6, distribuídos de acordo com as mesorregiões catarinenses, finalizando-os com a criação de tabelas e dos mapas dos clusters de COVID-19 detectados em Santa Catarina.

Por se tratar da utilização de dados secundários e de acesso livre, não foi necessária a aprovação do estudo em Comitê de Ética em Pesquisa (CEP). 


\section{Resultados}

\section{Análise descritiva}

O Estado de Santa Catarina registrou, entre 1o de março de 2020 e 31 de agosto de 2020, o total de 177.852 infectados pela COVID-19. Em números absolutos, a mesorregião do Vale do Itajaí apresentou a maior taxa de incidência por 100 mil habitantes do estado. No lado oposto, a mesorregião Serrana apontou a menor taxa de incidência por 100 mil habitantes. Nota-se que o Sul Catarinense também apresentou índices elevados próximos do Vale do Itajaí (Tabela 1).

A curva de evolução temporal de casos aumentou a partir do dia 29 de abril de 2020 e com maior incidência desde 29 de maio de 2020 (Figura 1), sendo que o ápice de novos infectados ocorreu em 13 de julho de 2020 no Vale do Itajaí e em 28 de julho de 2020 nas demais mesorregiões. A partir de

\section{Tabela 1}

Indicadores da COVID-19 por mesorregião catarinense. Santa Catarina, Brasil, de $1^{\circ}$ de março de 2020 a

31 de agosto de 2020 .

\begin{tabular}{lccc}
\hline Mesorregião catarinense & Número de casos & $\begin{array}{c}\text { Número de } \\
\text { habitantes }\end{array}$ & $\begin{array}{c}\text { Taxa de incidência } \\
\text { (\% por 100 mil habitantes) }\end{array}$ \\
\hline Grande Florianópolis & 30.975 & 1.205 .756 & $2.568,93$ \\
Norte Catarinense & 28.073 & 1.421 .656 & $1.974,67$ \\
Oeste Catarinense & 28.268 & 1.302 .165 & $2.170,85$ \\
Serrana & 7.759 & 415.579 & $1.867,03$ \\
Sul Catarinense & 29.628 & 1.042 .285 & $2.842,60$ \\
Vale do Itajaí & 53.149 & 1.865 .061 & $2.849,72$ \\
Total & 177.852 & 7.252 .502 & $2.452,28$ \\
\hline
\end{tabular}

\section{Figura 1}

Evolução dos casos de COVID-19 distribuídos por mesorregião catarinense. Santa Catarina, Brasil, 2000.

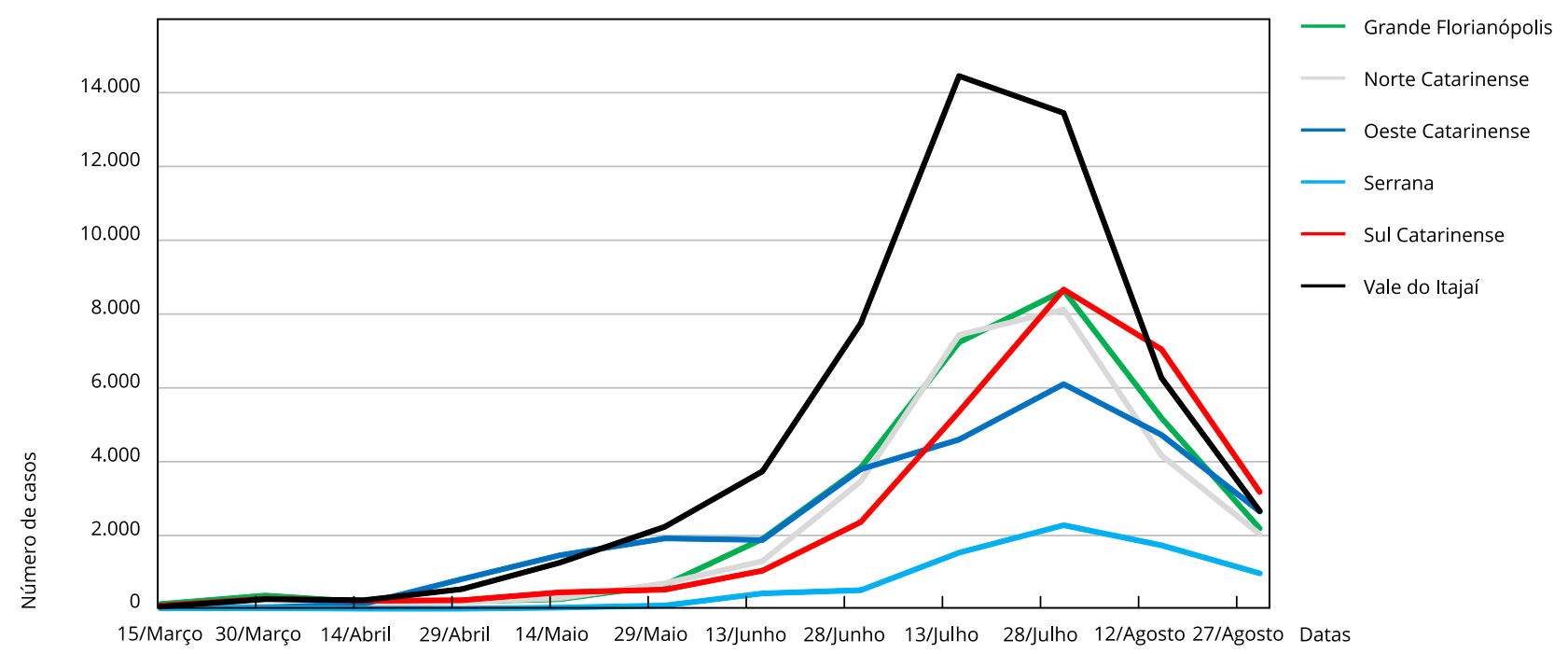


então, a curva de infectados apresentou queda brusca até o fim do intervalo analisado. Nessa época, o Sul Catarinense superou o Vale do Itajaí em novas notificações. Os registros da mesorregião Serrana sempre foram os mais moderados.

\section{Análise prospectiva sem covariável}

A estatística scan sem covariável identificou 17 agrupamentos ativos significativos $(\mathrm{p}<0,05)$ até a data 31 de agosto de 2020 (Tabela 2). O cluster primário (P1) teve centroide no sul catarinense (Figura 2), com RVG de 10.316,84 e raio de 48,44km. Nessa área, no prazo de 119 dias ocorreram 15.519 infecções por COVID-19. Sob a hipótese nula, de que não existisse agrupamento, seriam esperados 3.865 casos. O valor de p para este aglomerado, obtido a partir de 999 simulações pelo método de Monte Carlo, foi igual a 1×10-17. Como o valor resultante é menor que 0,05, a hipótese nula da não existência do cluster primário foi rejeitada, o que indica que o valor foi significativo. Os agrupamentos secundários foram analisados de modo análogo ao primário. Em relação ao início dos agrupamentos, constatou-se que todos conglomerados se originaram no mês de maio.

Tabela 2

Conglomerados de COVID-19 detectados pela análise prospectiva. Santa Catarina, Brasil, de 1o de março de 2020 a 31 de agosto de 2020 .

\begin{tabular}{|c|c|c|c|c|c|c|c|c|}
\hline Cluster & Mês de início & Raio (km) & Casos observados & Casos esperados & RVG & Valor de $p$ & Covariável & Centroide \\
\hline P1 & Maio/2020 & 48,44 & 15.519 & 3.865 & $10.316,84$ & $1,00 \mathrm{E}-17$ & Não & Sul Catarinense \\
\hline $\mathrm{S} 2$ * & Maio/2020 & 47,93 & 5.609 & 404 & $9.630,36$ & $1,00 \mathrm{E}-17$ & Não & $\begin{array}{c}\text { Oeste } \\
\text { Catarinense }\end{array}$ \\
\hline S3 & Maio/2020 & 48,91 & 7.967 & 1.113 & $8.963,08$ & $1,00 \mathrm{E}-17$ & Não & $\begin{array}{c}\text { Oeste } \\
\text { Catarinense }\end{array}$ \\
\hline S4 & Maio/2020 & 22,76 & 12.755 & 3.694 & $6.986,11$ & $1,00 \mathrm{E}-17$ & Não & $\begin{array}{c}\text { Grande } \\
\text { Florianópolis }\end{array}$ \\
\hline S5 & Maio/2020 & 48,97 & 7.888 & 1.473 & $6.941,20$ & $1,00 E^{-17}$ & Não & Vale do Itajaí \\
\hline s6 & Maio/2020 & 38,13 & 11.093 & 3.020 & $6.548,51$ & $1,00 E^{-17}$ & Não & Vale do Itajaí \\
\hline $\mathrm{S} 7$ * & Maio/2020 & 45,63 & 6.403 & 1.094 & $6.085,91$ & $1,00 E^{-17}$ & Não & Sul Catarinense \\
\hline S8 & Maio/2020 & 49,06 & 2.216 & 264 & $2.772,66$ & $1,00 E^{-17}$ & Não & Serrana \\
\hline S9 & Maio/2020 & 17,16 & 1.053 & 94 & $1.586,97$ & $1,00 \mathrm{E}-17$ & Não & $\begin{array}{c}\text { Grande } \\
\text { Florianópolis }\end{array}$ \\
\hline $\mathrm{S} 10$ * & Maio/2020 & 30,58 & 851 & 55 & $1.529,84$ & $1,00 E^{-17}$ & Não & $\begin{array}{c}\text { Oeste } \\
\text { Catarinense }\end{array}$ \\
\hline S11 & Maio/2020 & 21,71 & 1.982 & 419 & $1.523,88$ & $1,00 E^{-17}$ & Não & $\begin{array}{c}\text { Norte } \\
\text { Catarinense }\end{array}$ \\
\hline $\mathrm{S} 12$ * & Maio/2020 & 48,71 & 997 & 160 & 988,73 & $1,00 \mathrm{E}-17$ & Não & $\begin{array}{c}\text { Norte } \\
\text { Catarinense }\end{array}$ \\
\hline $\mathrm{S} 13$ * & Maio/2020 & 23,58 & 1.048 & 308 & 545,93 & $1,00 E^{-17}$ & Não & $\begin{array}{c}\text { Norte } \\
\text { Catarinense }\end{array}$ \\
\hline S14 & Maio/2020 & 37,64 & 320 & 34 & 428,71 & $1,00 \mathrm{E}-17$ & Não & Serrana \\
\hline S15 & Maio/2020 & 0,01 & 275 & 33 & 343,96 & $1,00 \mathrm{E}-17$ & Não & $\begin{array}{c}\text { Norte } \\
\text { Catarinense }\end{array}$ \\
\hline S16 & Maio/2020 & 49,34 & 820 & 288 & 325,96 & $1,00 E^{-17}$ & Não & $\begin{array}{c}\text { Norte } \\
\text { Catarinense }\end{array}$ \\
\hline
\end{tabular}

(continua) 
Tabela 2 (continuação)

\begin{tabular}{|c|c|c|c|c|c|c|c|c|}
\hline Cluster & Mês de início & Raio (km) & Casos observados & Casos esperados & RVG & Valor de $p$ & Covariável & Centroide \\
\hline S17 * & Maio/2020 & 41,75 & 2.981 & 2.445 & 55,73 & $1,00 \mathrm{E}-17$ & Não & Serrana \\
\hline P1 & Maio/2020 & 48,44 & 15.519 & 4.676 & $8.121,55$ & $1,00 \mathrm{E}-17$ & Sim & Sul Catarinense \\
\hline \multirow[t]{2}{*}{ S2 } & Maio/2020 & 22,76 & 12.755 & 3.537 & $7.389,01$ & $1,00 \mathrm{E}-17$ & Sim & Grande \\
\hline & & & & & & & & Florianópolis \\
\hline \multirow[t]{2}{*}{ S3 } & Maio/2020 & 49,78 & 4.650 & 408 & $7.119,23$ & $1,00 \mathrm{E}-17$ & Sim & Oeste \\
\hline & & & & & & & & Catarinense \\
\hline \multirow[t]{2}{*}{ S4 } & Maio/2020 & 48,91 & 7.967 & 1.603 & $6.526,29$ & $1,00 \mathrm{E}^{-17}$ & Sim & Oeste \\
\hline & & & & & & & & Catarinense \\
\hline S5 & Maio/2020 & 38,13 & 11.093 & 3.085 & $6.375,81$ & $1,00 \mathrm{E}^{-17}$ & Sim & Vale do Itajaí \\
\hline S6 & Maio/2020 & 48,97 & 7.888 & 1.723 & $5.943,46$ & $1,00 \mathrm{E}-17$ & Sim & Vale do Itajaí \\
\hline S7 & Maio/2020 & 40,64 & 3.560 & 472 & $4.133,08$ & $1,00 \mathrm{E}-17$ & Sim & Sul Catarinense \\
\hline \multirow[t]{2}{*}{ S8 } & Maio/2020 & 17,16 & 1.053 & 65 & $1.946,96$ & $1,00 \mathrm{E}-17$ & Sim & Grande \\
\hline & & & & & & & & Florianópolis \\
\hline S9 & Maio/2020 & 49,06 & 2.216 & 420 & $1.897,55$ & $1,00 E^{-17}$ & Sim & Serrana \\
\hline \multirow[t]{2}{*}{ S10 } & Maio/2020 & 27,19 & 1.949 & 373 & $1.654,11$ & $1,00 \mathrm{E}^{-17}$ & Sim & Oeste \\
\hline & & & & & & & & Catarinense \\
\hline \multirow[t]{2}{*}{ S11 } & Maio/2020 & 21,71 & 1.982 & 447 & $1.422,14$ & $1,00 \mathrm{E}-17$ & Sim & Norte \\
\hline & & & & & & & & Catarinense \\
\hline \multirow[t]{2}{*}{ S12 } & Maio/2020 & 29,44 & 868 & 165 & 737,96 & $1,00 \mathrm{E}-17$ & Sim & Norte \\
\hline & & & & & & & & Catarinense \\
\hline \multirow[t]{2}{*}{ S13 } & Maio/2020 & 43,93 & 589 & 91 & 600,01 & $1,00 \mathrm{E}-17$ & Sim & Norte \\
\hline & & & & & & & & Catarinense \\
\hline \multirow[t]{2}{*}{ S14 } & Maio/2020 & 0,00 & 275 & 33 & 337,71 & $1,00 \mathrm{E}-17$ & Sim & Norte \\
\hline & & & & & & & & Catarinense \\
\hline S15 & Maio/2020 & 37,64 & 320 & 53 & 309,47 & $1,00 \mathrm{E}^{-17}$ & Sim & Serrana \\
\hline \multirow[t]{2}{*}{ S16 ** } & Maio/2020 & 0,00 & 138 & 14 & 193,27 & $1,00 \mathrm{E}^{-17}$ & Sim & Norte \\
\hline & & & & & & & & Catarinense \\
\hline \multirow[t]{2}{*}{ S17 } & Maio/2020 & 49,34 & 820 & 413 & 155,47 & $1,00 \mathrm{E}-17$ & Sim & Norte \\
\hline & & & & & & & & Catarinense \\
\hline S18 & Julho/2020 & 0,01 & 10 & 0 & 29,83 & $3,23 E-10$ & Sim & Serrana \\
\hline
\end{tabular}

RVG: razão de verossimilhança generalizada de Poisson.

* Clusters explicados parcialmente pela inclusão da covariável tendência de mobilidade dos locais de trabalho;

** Novo cluster detectado após a inclusão da covariável tendência de mobilidade dos locais de trabalho.

Houve a abrangência de agrupamentos espaço-temporais de alto risco registrados por COVID-19 em todas as mesorregiões catarinenses (Figura 2). Os três principais clusters sem covariáveis ficaram centrados no Sul (um) e Oeste Catarinense (dois), respectivamente.

O agrupamento $\mathrm{S} 15$, situado no Norte Catarinense, tem raio de apenas 0,01km. Contudo sabe-se que, na prática, sua abrangência é maior. Essa particularidade aconteceu por causa do georreferenciamento dos casos estarem centrados por município. Todavia, salienta-se que o tamanho do raio maior $(50 \mathrm{~km})$ não está relacionado necessariamente com a importância.

\section{Análise prospectiva com a covariável tendência de mobilidade dos locais de trabalho}

Quando associada com a covariável tendência de mobilidade dos locais de trabalho, a análise prospectiva detectou 18 conglomerados significativos $(\mathrm{p}<0,05)$ (Tabela 2). Em comparação com os agrupamentos localizados apenas com a variável, observou-se que seis clusters são explicados em parte pela tendência de mobilidade dos locais de trabalho e mais um que não havia sido encontrado anteriormente (Figura 3). Outro fato interessante foi a alteração da ordem dos aglomerados secundários. Logo, há diferenças na deteç̧ão em relação ao modelo sem essa covariável. 
Figura 2

Mapa dos clusters de COVID-19 detectados pela análise prospectiva. Santa Catarina, Brasil, de 1 o de março de 2020 a 31 de agosto de 2020.

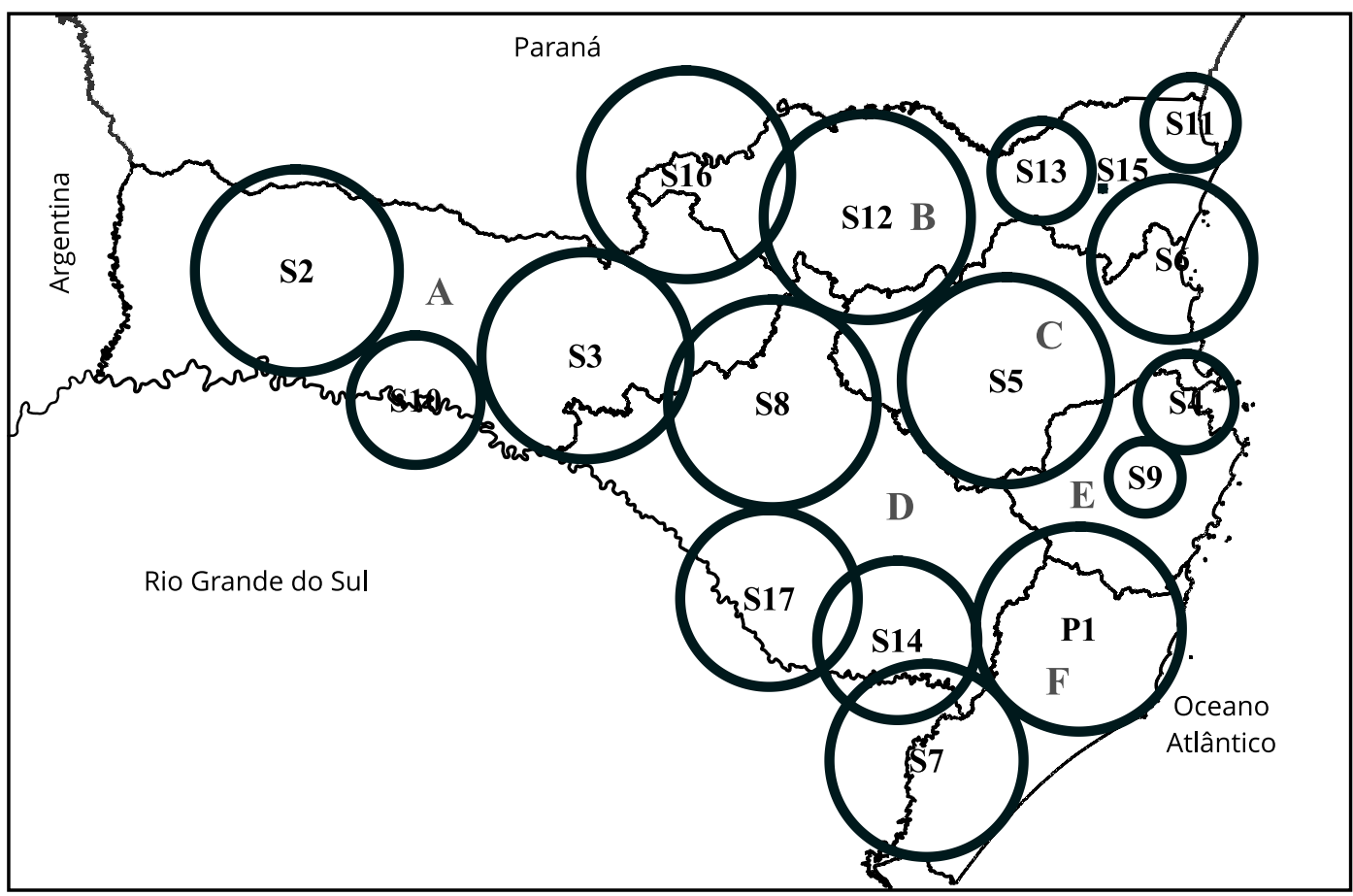

Raio do cluster sem covariáve
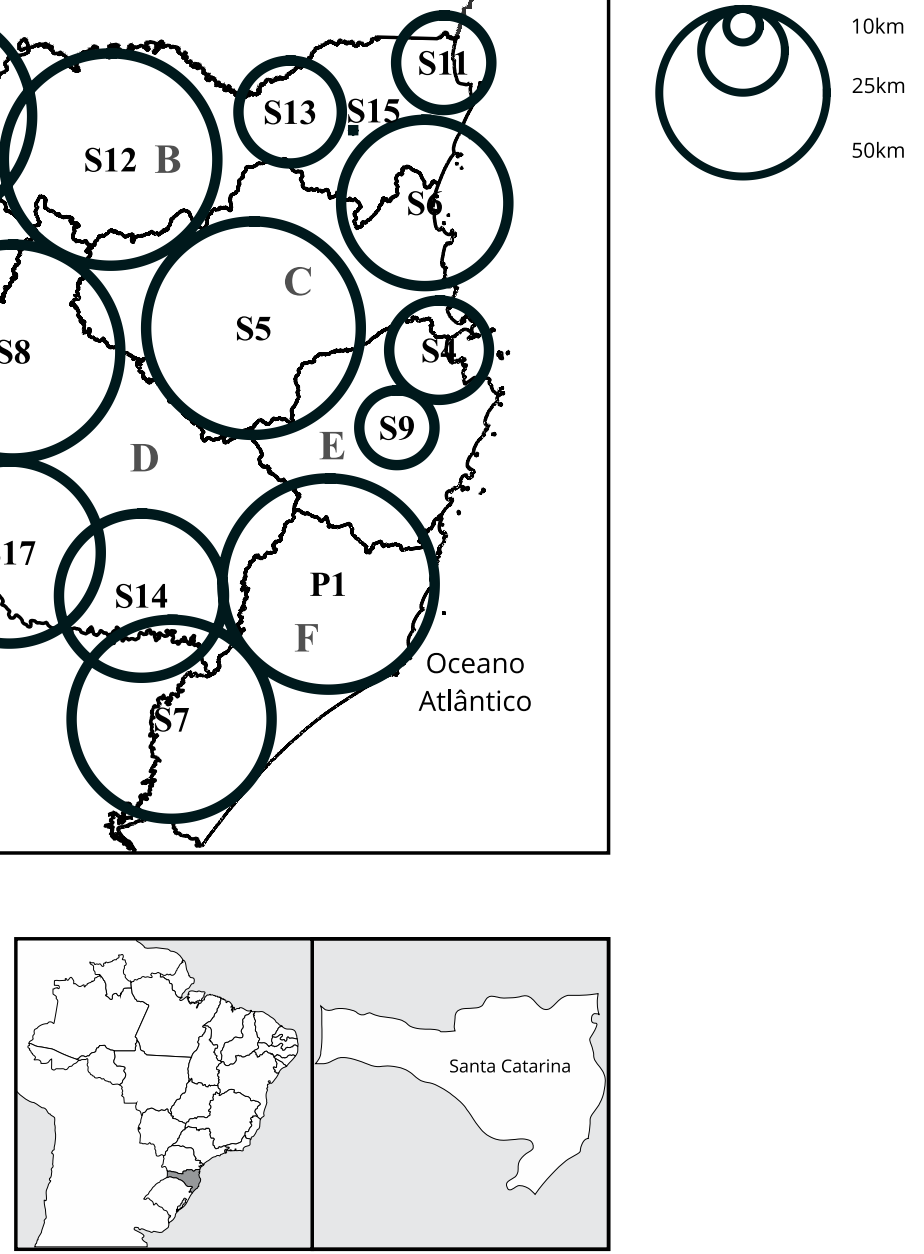

A: Oeste Catarinense; B: Norte Catarinense; C: Vale do Itajái; D: Serrana; E: Grande Florianópolis; F: Sul Catarinense; P1: hierarquização do cluster. Fontes: dados COVID-19 (Portal de Dados Abertos do Estado de Santa Catarina. Dados anonimizados: casos confirmados COVID-19.

http://www.dados.sc.gov.br/dataset/covid-19-dados-anonimizados-de-casos-confirmados/resource/76d6dfe8-7fe9-45c1-95f4-cab971803d49, acessado em 04/Set/2020); dados vetoriais (Instituto Brasileiro de Geografia e Estatística. Estimativas de população 2020. https://www.ibge.gov.br/estatisticas/ sociais/populacao/9103-estimativas-de-populacao.html?=\&t=downloads, acessado em 22/Ago/2020); datum horizontal (Instituto Brasileiro de Geografia e Estatística. Malha municipal. Downloads: municipio_2019. https://www.ibge.gov.br/geociencias/organizacao-do-territorio/15774-malhas. html?=\&t=downloads, acessado em 22/Ago/2020). 
Figura 3

Mapa dos clusters de COVID-19 detectados pela análise prospectiva, considerando a covariável tendência de mobilidade dos locais de trabalho. Santa Catarina, Brasil, de 1 o de março de 2020 a 31 de agosto de 2020.

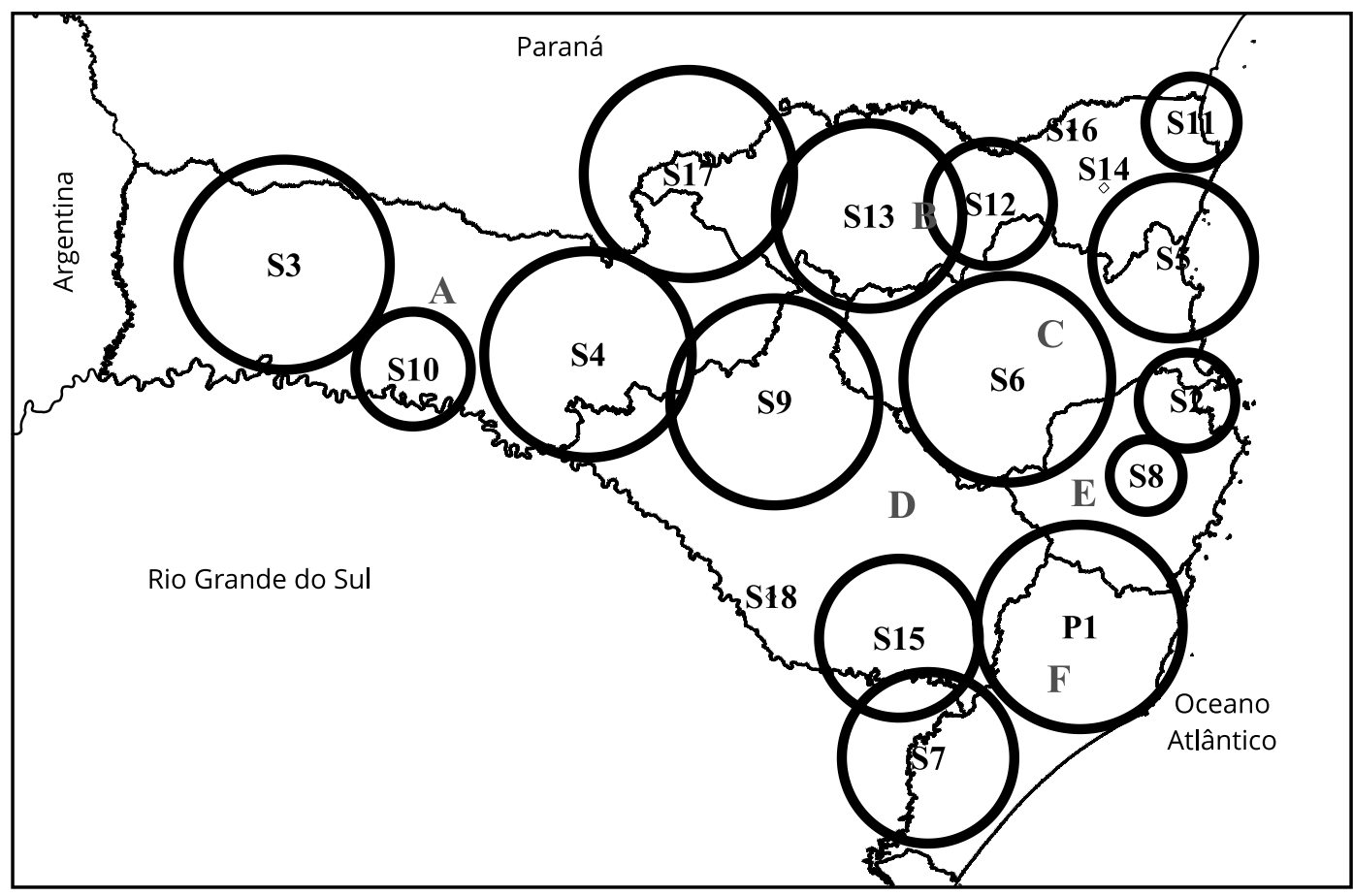

Raio do cluster com covariável
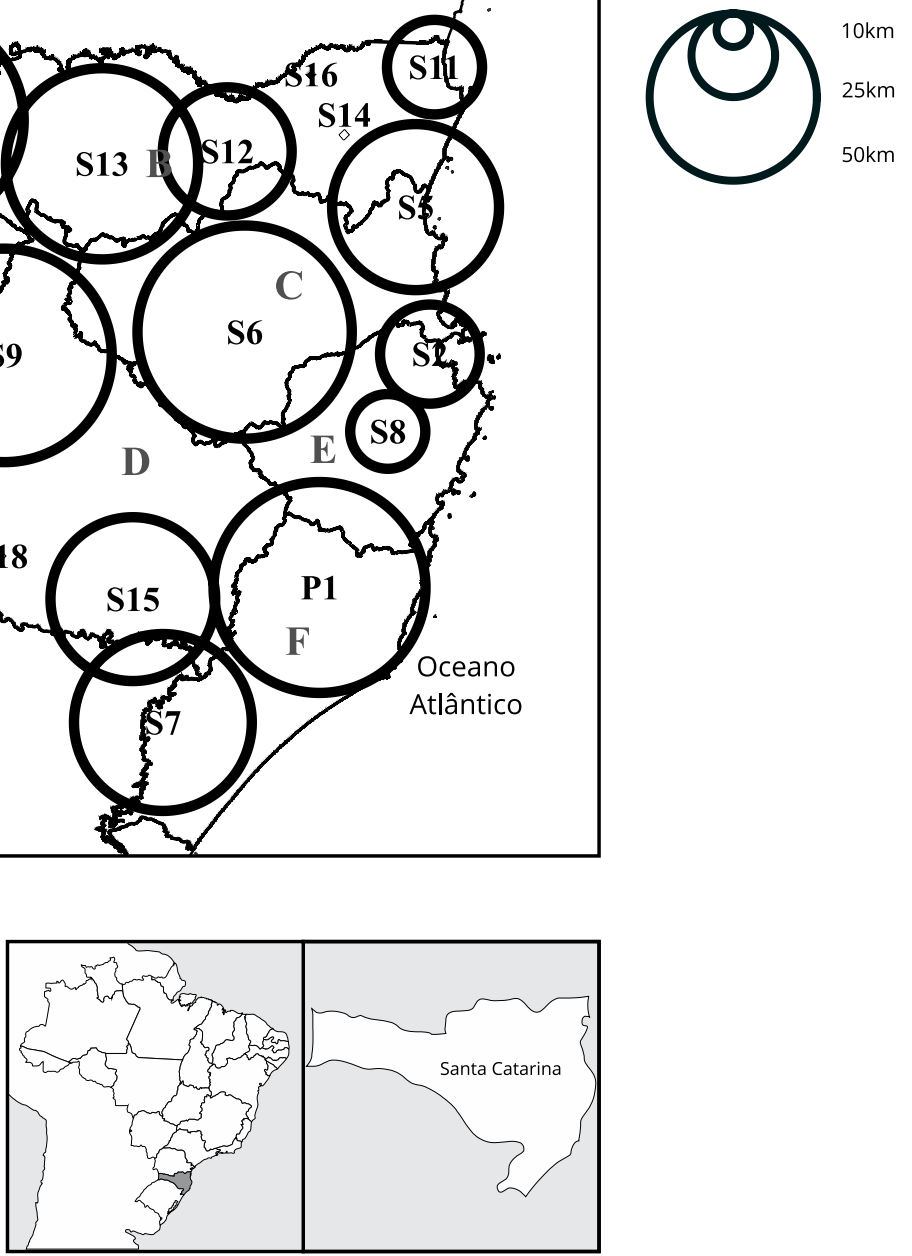

A: Oeste catarinense; B: Norte catarinense; C: Vale do Itajái; D: Serrana; E: Grande Florianópolis; F: Sul catarinense; P1: hierarquização do cluster. Fontes: dados COVID-19 (Portal de Dados Abertos do Estado de Santa Catarina. Dados anonimizados: casos confirmados COVID-19. http://www.dados. sc.gov.br/dataset/covid-19-dados-anonimizados-de-casos-confirmados/resource/76d6dfe8-7fe9-45c1-95f4-cab971803d49, acessado em 04/Set/2020); taxa relativa de comparecimento ao local de trabalho (COVID-19: relatórios de mobilidade da comunidade. https://support.google.com/covid19mobility/answer/9824897?hl=pt-BR\&ref_topic=9822927, acessado em 22/Ago/2020) 
Dentre as principais diferenças constatadas, salienta-se a mudança do aglomerado secundário (S2) para a mesorregião da Grande Florianópolis. Com isso, os três principais clusters ficaram centrados nas mesorregiões Sul, Grande Florianópolis e Oeste Catarinense, respectivamente.

O conglomerado S18 começou no mês de julho de 2020, os demais permaneceram com início no mês de maio de 2020. Os agrupamentos S14 e S16 tiveram raios com comprimentos insignificantes, diferente do que acontece na realidade.

\section{Discussão}

A distribuição espaço-temporal dos clusters de COVID-19 em Santa Catarina indicou a presença de infectados em todas as mesorregiões do estado. Pesquisas nos estágios iniciais apontaram a maior suscetibilidade a SARS-CoV-2 no litoral e no Oeste Catarinense 16,17. De fato, essa característica se manteve nas etapas seguintes, sendo que os agrupamentos prioritários de intervenção até o fim da pesquisa ficaram situados no sul catarinense, oeste catarinense, na Grande Florianópolis e no Vale do Itajaí.

Estima-se que a dispersão da doença para os outros locais de Santa Catarina ocorreu através da Rodovia BR-101, situada no litoral, e das Rodovias BR-153 e BR-282, no Oeste Catarinense, que são os principais eixos rodoviários do estado e têm intenso fluxo de pessoas e mercadorias.

A curva de crescimento da COVID-19 em Santa Catarina ocorreu entre maio a julho de 2020, com ápice na segunda quinzena de julho. Dentre os fatores associados ao pico de contágio, pode-se citar: a liberação do transporte coletivo intermunicipal no início de junho 18 e a redução na adesão ao distanciamento social 19,20 por parte dos catarinenses. O início do inverno catarinense foi outro fator agravante dessa situação, pois o SARS-Cov 2 tem maior capacidade de transmissão sob clima mais frio e seco 21.

O trabalho remoto é uma medida recomendada para o distanciamento social. Quando esse critério não é adotado, a presença de funcionários nos espaços físicos das unidades empregadoras é maior, ou seja, a tendência de mobilidade dos locais de trabalho é elevada. A determinação do índice de distanciamento social é uma grandeza de difícil aquisição, principalmente em níveis regionais, diferentemente da tendência de mobilidade dos locais de trabalho, que apresentava a série de dados mais completa. Portanto, a influência dessa covariável no estudo pode ser um indicativo da capacidade do distanciamento social em conter a disseminação da doença conforme sugere a literatura 22,23,24.

Na comparação entre os resultados obtidos e o contexto estadual na disseminação da COVID-19, observou-se a importância da estatística scan para estudos epidemiológicos, uma vez que o agrupamento prioritário ativo ficou situado no Sul Catarinense, mesorregião que apresentava a maior quantidade de novos casos até 31 de agosto de 2020, data final da pesquisa.

A estatística scan é uma ferramenta poderosa para a vigilância epidemiológica, pois pode auxiliar na tomada de decisão referente à pandemia no país 12 . A análise espacial de uma nova doença é essencial para a compreensão de padrões e adoção de medidas profiláticas 25 .

\section{Considerações finais}

O uso da estatística scan no estudo da COVID-19 em Santa Catarina demonstrou o crescimento intenso no contágio por SARS-CoV-2 entre maio a julho de 2020. Após esse período, houve decréscimo do número de novos casos diários, porém insuficiente para evitar a formação de clusters da doença e reduzir os impactos da epidemia. Entretanto, ao abordar essa redução, é preciso ter muita cautela, pois a subnotificação de casos pode proporcionar uma falsa percepção de diminuição do contágio.

A COVID-19 estava difundida em todas as mesorregiões catarinenses, com maior intensidade no Sul Catarinense, Oeste Catarinense e na Grande Florianópolis. Nesse cenário, este artigo atingiu o objetivo de aplicar a estatística scan para identificar os conglomerados ativos de COVID-19 no estado ao nível de 5\% de significância, classificando-os conforme prioridade de intervenção.

A importância das medidas de distanciamento social para redução do contágio do coronavírus já foi investigada em diversas pesquisas e apresentou evidências relevantes para o Estado de Santa Cata- 
rina, visto que, após a inclusão da covariável tendência de mobilidade dos locais de trabalho, houve a explicação moderada de 38,89\% dos agrupamentos de COVID-19.

Considerando que o principal modo de contenção da doença ainda é por meio do isolamento social e consequente quebra do ciclo de transmissão, a análise espaço-temporal é primordial para compreender padrões e executar medidas de intervenção.

Algumas limitações são importantes para a interpretação dos resultados encontrados. Primeiramente, a base de dados sobre a tendência de mobilidade dos locais de trabalho apresentava dados faltantes e que foram repostos com médias estaduais. A população de referência é uma estimativa do IBGE, o que pode gerar pequenas diferenças na detecção dos aglomerados.

Em trabalhos futuros, sugere-se a inclusão de novos critérios do distanciamento social no modelo para averiguar sua influência nos conglomerados. O aperfeiçoamento do modelo com essas novas medidas deve gerar resultados mais próximos com a realidade local.

\section{Colaboradores}

I. Merêncio foi responsável pela concepção da ideia do artigo, curadoria dos dados, análise formal, redação inicial, revisão e edição, e aprovação da versão final. G. M. Monteiro foi responsável pela curadoria dos dados, análise formal, redação inicial, revisão e edição, e aprovação da versão final. C. A. O. Vieira executou a análise formal, a supervisão, a revisão e edição, e aprovação da versão final.

\section{Informações adicionais}

ORCID: Ivan Merêncio (0000-0003-1288-124X); Gecielli Martins Monteiro (0000-0001-9470193X); Carlos Antônio Oliveira Vieira (0000-00034047-8466).

\section{Referências}

1. Ren L, Wang Y, Wu Z, Xiang Z, Guo L, Xu T, et al. Identification of a novel coronavirus causing severe pneumonia in human. Chin Med J 2017; 133:1015-24.

2. Huang C, Wang Y, Li X, Ren L, Zhao J, Hu Y, et al. Clinical features of patients infected with 2019 novel coronavirus in Wuhan, China. Lancet 2020; 395:497-506.

3. Adhikari SP, Meng S, Wu Y, Mao Y, Ye R, Wan $\mathrm{Q}$, et al. Epidemiology, causes, clinical manifestation and diagnosis, prevention and control of coronavirus disease (COVID-19) during the early outbreak period: a scoping review. Infect Dis Poverty 2020; 9:29.

4. Lakhani A. Which Melbourne metropolitan areas are vulnerable to COVID-19 based on age, disability, and access to health services? Using spatial analysis to identify service gaps and inform delivery. J Pain Symptom Manage 2020; 60:41-4.

5. Liu Y, Gayle AA, Wilder-Smith A, Rocklöv J. The reproductive number of COVID-19 is higher compared to SARS coronavirus. J Travel Med 2020; 27:1015-24.

6. Hamid S, Mir MY, Rohela GK. Novel coronavirus disease (COVID-19): a pandemic (epidemiology, pathogenesis and potential therapeutics). New Microbe and New Infect 2020; $35: 100679$. 
7. Secretaria de Vigilância em Saúde, Ministério da Saúde. Boletim Epidemiológico Especial Doença pelo Coronavírus COVID-19: semana epidemiológica 35 (23 a 29/08) 2020; (29). https://antigo.saude.gov.br/images/pdf/2020/ September/02/18h-Boletim-epidemiologicoCOVID-29-final.pdf.

8. Boulos MNK, Geraghty EM. Geographical tracking and mapping of coronavirus disease COVID-19/severe acute respiratory syndrome coronavirus 2 (SARS-CoV-2) epidemic and associated events around the world: how 21st century GIS technologies are supporting the global fight against outbreaks and epidemics. Int J Health Geogr 2020; 19:8.

9. Araújo TC. Extensão da estatística Scan para detecção de conglomerados espaço-temporais em dados com excesso de zeros [Dissertação de Mestrado]. Brasília: Universidade de Brasília; 2012.

10. Rangel MES. Dinâmica espacial e contingências socioambientais da hanseníase no estado do Maranhão: avaliação de riscos e vulnerabilidades em áreas hiperendêmicas [Tese de Doutorado]. São Paulo: Universidade de São Paulo; 2016.

11. Kaqui RL. Uma estatística de varredura espacial para dados de contagem com censura [Dissertação de Mestrado]. Brasília: Universidade de Brasília; 2016.

12. Barrozo LV, Serafim MB, Moraes SL, Mansur G. Monitoramento espaço-temporal das áreas de alto risco de COVID-19 nos municípios do Brasil. Hygeia (Uberlândia) 2020; (Edição Especial: COVID-19):417-25.

13. Lau H, Khosrawipour V, Kocbach P, Mikolajczyk A, Schubert J, Bania J, et al. The positive impact of lockdown in Wuhan on containing the COVID-19 outbreak in China. J Travel Med 2020; 3:18-27.

14. Kulldorff M, Heffernan R, Hartman J, Assunção R, Mostashari F. A space-time permutation scan statistic for disease outbreak detection. PLoS Med 2005; 2:216-24.

15. Vieira CAO, Santos NT, Carneiro APS, Balieiro AAS. Brazilian Amazonia deforestation detection using spatio-temporal scan statistics. ISPRS Annals of Photogrammetry, Remote Sensing and Spatial Information Sciences. In: XXII ISPRS Congress. https://www.isprs. org/documents/archive/Volume-A/2012-Mel bourne/ (acessado em 22/Ago/2020).

16. Vianna LFN, Furlanetti TR, Luna MMM. Índice de vulnerabilidade social à COVID-19 por setor censitário e por município em Santa Catarina. Florianópolis: Centro de Informações de Recursos Ambientais e de Hidrometeorologia de Santa Catarina; 2020.
17. Nascimento Júnior L, Ribeiro EAW, Menegon FA, Springer KS, Monguilhot M, Meliani PF. Suscetibilidade à COVID-19 em Santa Catarina: uma proposta metodológica. Hygeia (Uberlândia) 2020; (Edição Especial: COVID-19):274-86.

18. Governo do Estado de Santa Catarina. Decreto no 630 , de 1o de junho de 2020. Altera o Decreto no 562, de 2020, que declara estado de calamidade pública em todo o território catarinense, nos termos do COBRADE no 1.5.1.1.0 - doenças infecciosas virais, para fins de enfrentamento à COVID-19, e estabelece outras providências. Diário Oficial do Estado de Santa Catarina 2020; 1 jun.

19. Farias HS. O avanço da COVID-19 e o isolamento social como estratégia para redução da vulnerabilidade. Espaço e Economia 2020; 17:1-12.

20. Camargo EB, Elias FTS. Nota rápida de evidência: o papel dos transportes públicos na transmissão de COVID-19. Brasília: Fundação Oswaldo Cruz; 2020.

21. Confalonieri U, Rangel E, Oliveira ML, Menezes J, Santos R. Coronavírus e clima. Climacon 2020; 7. http://climacom.mudancasclimaticas. net.br/ulisses-confalonieri-elizabeth-rangel -maria-de-lourdes-oliveira-julia-menezes-e -rhavena-santos-coronavirus-e-clima/.

22. Aquino EML, Silveira IH, Pescarini JM, Aquino R, Souza-Filho JA, Rocha AS, et al. Medidas de distanciamento social no controle da pandemia de COVID-19: potenciais impactos e desafios no Brasil. Ciênc Saúde Colet 2020; 25:2423-46.

23. Ganem F, Mendes FM, Oliveira SB, Porto VB, Araújo W, Nakaya $\mathrm{H}$, et al. The impact of early social distancing at COVID-19 outbreak in the largest Metropolitan Area of Brazil. medRxiv 2020; 15 abr. https://www.medrxiv.org/conte $\mathrm{nt} / 10.1101 / 2020.04 .06 .20055103 \mathrm{v} 2$.

24. Hou J, Hong J, Ji B, Dong B, Chen Y, Ward $\mathrm{MP}$, et al. Changing transmission dynamics of COVID-19 in China: a nationwide population-based piecewise mathematical modelling study. medRxiv 2020; 30 mar. https://www. medrxiv.org/content/10.1101/2020.03.27.200 $45757 \mathrm{v} 1$.

25. Cavalcante JR, Abreu AJL. COVID-19 no município do Rio de Janeiro: análise espacial da ocorrência dos primeiros casos e óbitos confirmados. Epidemiol Serv Saúde 2020; 29:e2020204. 


\section{Abstract}

The application of spatial analysis to the study of human epidemiological data has gained great momentum in the last two decades. This article approaches the scan statistic for the detection of spatial-temporal clusters of COVID-19 cases in the State of Santa Catarina, Brazil. The aim is to apply the scan statistic to identify active clusters, determining their location, size, and order (priority). Organization of the descriptive based included COVID-19 cases from March 1 to August 31, 2020, available in the Santa Catarina State Open Data Portal. The vector base of the municipal limits and mesoregions in Santa Catarina and the population estimates for 2020 were obtained from the Brazilian Institute of Geography and Statistics (IBGE) website. The workplace mobility trend covariable was obtained from the document COVID-19: Report on Community Mobility in Google. Execution of the statistic considered the discrete Poisson model, supported by the prospective approach. The study's results evidenced the procedure's capacity to demarcate clusters, identifying 17 active clusters with the response variable and 18 active clusters after inclusion of the covariable, distributed throughout the state and predominantly on the coast and the Western region. The primary cluster was in Southern Santa Catarina. The workplace mobility trend covariable moderately influenced $38.89 \%$ of the clusters. The method proved to be efficient for understanding the epidemic's spatial distribution. This characterizes the scan statistic as a tool to support the execution of actions by policymakers, prioritizing areas most affected by the disease.

Coronavirus; Medical Geography; SpatioTemporal Analysis

\section{Resumen}

La aplicación del análisis espacial, destinado al estudio de datos epidemiológicos humanos, se hizo notoria en las últimas dos décadas. En este sentido, este artículo aborda la estadística scan para la detección de clústeres espacio-temporales de casos de COVID-19 en Santa Catarina, Brasil. El objetivo es aplicar la estadística scan para la identificación de agrupamientos activos, determinando su localización, dimensión y orden (prioridad). La organización de la base descriptiva abarcó los casos de COVID-19 entre el 01 de marzo a 31 de agosto de 2020, disponibles en el Portal de Datos Abiertos del Estado de Santa Catarina. La base vectorial de los limites municipales y mesorregiones catarinenses, $y$ las poblaciones estimadas para 2020, se obtuvieron en el sitio web del Instituto Brasileño de Geografía y Estadística (IBGE). La covariable tendencia de movilidad de los lugares de trabajo se obtuvo en el documento COVID-19: Informe de Movilidad de la Comunidad de Google. Para la ejecución de la estadística se consideró el modelo discreto de Poisson, apoyado en el abordaje prospectivo. En el resultado del trabajo se evidenció la capacidad del procedimiento para la delimitación de los clústeres, lo cual identificó 17 clústeres activos con la variable respuesta y 18 clústeres activos tras la inclusión de la covariable, distribuidos en todo el estado, predominantes en la costa y en el Oeste Catarinense. El clúster primario se localizó en el Sur Catarinense. La covariable tendencia de movilidad de los lugares de trabajo influenció moderadamente en un 38,89\% de los aglomerados. El método fue eficiente para la comprensión de la distribución espacial de la epidemia. Esto caracteriza la estadística scan como una herramienta de apoyo para la ejecución de acciones que deben ser tomadas por gestores, priorizando las áreas más afectadas por la enfermedad.

Coronavirus; Geografía Médica; Análisis

Espacio-Temporal
Recebido em 18/Out/2020

Versão final reapresentada em 09/Mar/2021

Aprovado em 26/Abr/2021 